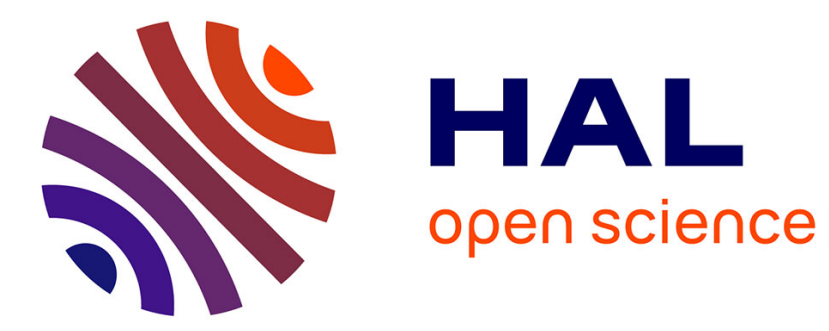

\title{
Search for exotic Higgs outside the MSSM
}

A. Tilquin

\section{- To cite this version:}

A. Tilquin. Search for exotic Higgs outside the MSSM. International Conference On Hadron Spectroscopy (HADRON 99) 8, Aug 1999, Beijing, China. pp.137-140. in2p3-00008199

\section{HAL Id: in2p3-00008199 https://hal.in2p3.fr/in2p3-00008199}

Submitted on 5 Feb 2001

HAL is a multi-disciplinary open access archive for the deposit and dissemination of scientific research documents, whether they are published or not. The documents may come from teaching and research institutions in France or abroad, or from public or private research centers.
L'archive ouverte pluridisciplinaire HAL, est destinée au dépôt et à la diffusion de documents scientifiques de niveau recherche, publiés ou non, émanant des établissements d'enseignement et de recherche français ou étrangers, des laboratoires publics ou privés. 


\title{
Search for Exotic Higgs Outside the MSSM
}

\author{
André Tilquin (Tilquin@cppm.in2p3.fr) a \\ ${ }^{a}$ Centre de Physique des Particules de Marseille, \\ CPPM, 163 Avenue de Luminy, Case 907, F-13288 Marseille
}

Recent preliminary results of searches for Higgs bosons outside the Minimal Supersymetric Standard Model (MSSM) are summarised. These results are based on data from the four LEP experiments: ALEPH, DELPHI, L3 and OPAL, at a centre-of-mass energies up to $189 \mathrm{GeV}$. Some new results from the two Tevatron experiments: CDF and D0, are also presented. Finally, the potential mass reach for exotic Higgs at future accelerators are discussed.

\section{Introduction}

At the present level of accuracy, the fundamental electro-week interactions are perfectly described by the Standard Model based on the $\mathrm{SU}(2) \otimes \mathrm{U}(1)$ gauge symmetry group. As a consequence of this gauge group all particles should be massless. To break down this symmetry, a scalar SU(2) Higgs doublet with a non-zero vacuum expectation value is introduced and spontaneously generates gauge boson masses. The fermion masses are obtained by adding a Yukawa coupling to the Higgs boson.

For a Higgs accessible at LEP energies, the small Yukawa coupling $\left(\approx 5.10^{-4}\right.$ for $\left.\mathrm{Hb} \overline{\mathrm{b}}\right)$ implies that any new particle interacting even weakly with the Standard Model particles and the Higgs can easily dominate over all possible Higgs decay modes, leading to an invisible Higgs.

The Yukawa coupling between the Higgs and the fermions is introduced to generate fermion masses with the same Higgs field. But there are no strong reasons to justify this choice and fermion masses may have nothing to do with the mass generation for bosons. In such a case, the Higgs only couples to gauge bosons and thus decays to two photons through a W loop. This special feature is described in the framework of two Higgs doublet models (2HDM).

More generally, in the 2HDM models, the physical Higgs spectrum contains five Higgs fields $\left(\mathrm{h}, \mathrm{H}, \mathrm{A}, \mathrm{H}^{ \pm}\right)$. Outside the MSSM, the charged Higgs mass is not constrained and might be low.

This review summarises the searches for invisible, fermiophobic and charged Higgs decays performed by the four LEP experiments at a maximum centre-of-mass energy of $189 \mathrm{GeV}$ with an integrated luminosity of approximately $175 \mathrm{pb}^{-1} /$ expt. and by the two Tevatron experiments with an integrated luminosity of approximately $100 \mathrm{pb}^{-1} /$ expt. 


\section{Invisible Higgs bosons decay}

In the context of generating neutrino mass, some extensions of the Standard Model called the Majoron models are characterised by the existence of a Golstone boson (the Majoron) coming from the spontaneous breaking of a new global U(1) symmetry. Since the coupling of the Majoron to the Higgs particle is not required to be small, the Higgs particle could predominantly decays into an invisible Majoron pair.

Invisible Higgs bosons decay may also occur in some SUSY models where the usually neglected mode $\mathrm{h} \rightarrow \chi_{1}^{0} \chi_{1}^{0}$ can dominates over the $\mathrm{h} \rightarrow \mathrm{b} \overline{\mathrm{b}}$ signal.

The topology of invisibly decaying Higgs boson produced through the process $\mathrm{e}^{+} \mathrm{e}^{-} \rightarrow \mathrm{hZ}$ is characterised by either two jets or two leptons from the $\mathrm{Z}^{0}$ decay, with large missing momentum and a visible mass of the event consistent with the $\mathrm{Z}$ mass. The main background is from semi-leptonic decays of $\mathrm{W}$ pairs and from $\mathrm{ZZ}$ events in which a $\mathrm{Z}$ decays in two neutrinos.

No excess of events from the invisible Higgs decay searches have been observed. The exclusion curve obtains by the OPAL experiment is shown in figure 1. Assuming a Standard Model cross section and an invisible Higgs decay branching ratio of 1 , a lower limit on the Higgs mass has been obtained by the four LEP experiments (Table 1). Assuming Standard Model decay probabilities in the visible part and combining with visible channels analyses, DELPHI gives an absolute lower limit on the Higgs mass independently of the invisible Higgs branching ratio of $91.1 \mathrm{GeV} / \mathrm{c}^{2}$ as shown in figure 2 .

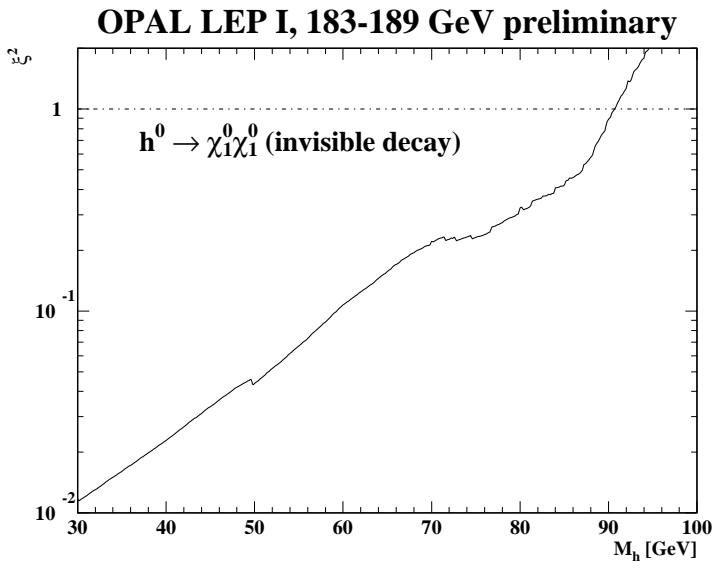

Figure 1. OPAL exclusion curve for invisible Higgs decays. The production cross section is expressed as $\xi^{2} \sigma_{\mathrm{SM}}\left(\mathrm{e}^{+} \mathrm{e}^{-} \rightarrow \mathrm{HZ}\right)$.

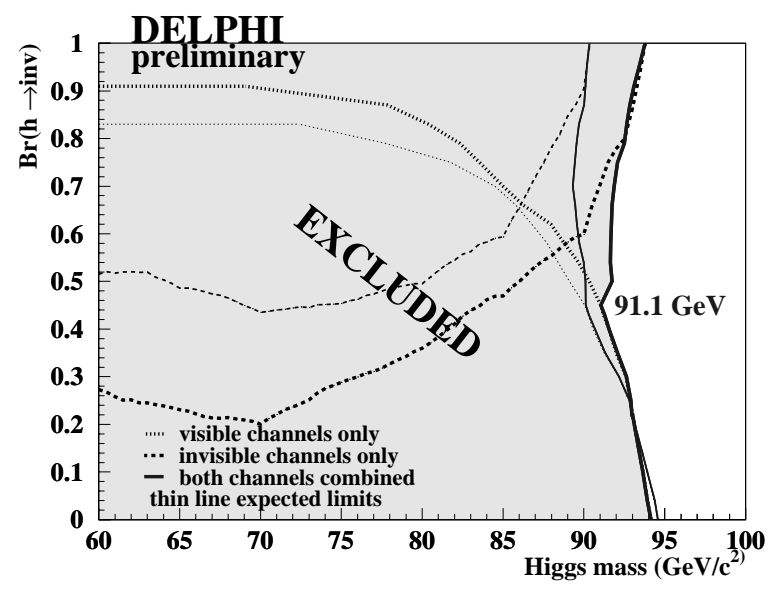

Figure 2. DELPHI results on invisible Higgs decay combined with visible Higgs decay analyses. 


\section{Fermiophobic Higgs bosons}

In minimal extensions of the Standard Model, two Higgs doublets are introduced in order to give masses to up-type quarks and down type quarks separately. In one of the four possible versions of these models, only the $\mathrm{H}_{2}$ Higgs doublets couples to fermions. The lighter of the two neutral CP-even Higgs bosons, $\mathrm{h}=\mathrm{H}_{1}^{0} \sin \alpha+\mathrm{H}_{2}^{0} \cos \alpha$, only couples to bosons if the mixing angle $\alpha$ is equal to $\pi / 2$. In this case, the production cross section $\mathrm{e}^{+} \mathrm{e}^{-} \rightarrow$ hZ has the Standard Model strength and the Higgs decay branching ratio in two photons, via a $\mathrm{W}$ loop, is dominant up to a Higgs mass of $90 \mathrm{GeV} / \mathrm{c}^{2}$.

In all LEP experiments, the $\nu \bar{\nu} \gamma \gamma ; \mathrm{l}^{+} \mathrm{l}^{-} \gamma \gamma$ and $\mathrm{q} \overline{\mathrm{q}} \gamma \gamma$ topologies have been studied. The main background is from initial state radiation and 4 fermions processes and is reduced by photon isolation criteria and kinematics cuts. In figure 3 the exclusion curve obtain by ALEPH and assuming a Standard Model production cross section is shown. Table 1 summarised the results from the four LEP experiments for the case of a fermiophobic Higgs branching ratio.

Similar analyses have been performed by CDF and D0 experiments using the associated productions: $\mathrm{qq}^{\prime} \rightarrow \mathrm{h}^{0} \mathrm{Z}(\mathrm{W})$, and the $\gamma \gamma q \overline{q^{\prime}}$ final state. With an integrated luminosity of about $100 \mathrm{pb}^{-1}$ a $95 \%$ C.L. lower limit on the fermiophobic Higgs mass of $82 \mathrm{GeV} / \mathrm{c}^{2}$ at $\mathrm{CDF}$ and $78.5 \mathrm{GeV} / \mathrm{c}^{2}$ at D0 have been set.

Table 1

LEP lower mass limits in $\mathrm{GeV} / \mathrm{c}^{2}$ at $95 \%$ C.L. for the Higgs boson decaying in different final states.

\begin{tabular}{lcccc}
\hline & ALEPH & DELPHI & L3 & OPAL \\
\hline $\mathrm{h}^{0} \rightarrow$ invisible & 95.4 & 93.8 & 95.0 & 90.6 \\
$\mathrm{~h}^{0} \rightarrow \gamma \gamma$ & 96.0 & 96.0 & 96.0 & 96.2 \\
$\mathrm{H}^{+} \mathrm{H}^{-}$ & 65.5 & 66.9 & 67.5 & 68.7 \\
\hline
\end{tabular}

\section{Charged Higgs bosons}

The charged Higgs boson is predicted in many extensions of the Standard Model. At LEP, it is pair produced through the mechanism: $\mathrm{e}^{+} \mathrm{e}^{-} \rightarrow \mathrm{H}^{+} \mathrm{H}^{-}$. In two Higgs doublets models the production cross section is completely specified at the Born level by the electric charge, the Weinberg angle $\theta_{\mathrm{W}}$ and the charged Higgs boson mass. The dominant Higgs branching ratios to the decay channels $\tau \overline{\nu_{\tau}}$ and $\mathrm{c} \overline{\mathrm{S}}$ are very model dependent. Assuming that $\mathrm{B}\left(\mathrm{H} \rightarrow \tau \overline{\nu_{\tau}}\right)+\mathrm{B}(\mathrm{H} \rightarrow \mathrm{c} \overline{\mathrm{S}})=1$, the analyses are performed in a model independent way.

In each LEP experiment, the three possible final states have been used: $\tau^{-} \overline{\nu_{\tau}} \tau^{+} \nu_{\tau}$, $\tau^{-} \overline{\nu_{\tau}} c \overline{\mathrm{s}}$ and $\mathrm{c} \overline{\mathrm{S}} \overline{\mathrm{C}} \mathrm{s}$. The analyses are based on four jet events or $\tau$ lepton identification with missing energy. No evidence for a signal is found. In Figure 4, mass limits are set as a function of the branching ratio $\mathrm{B}\left(\mathrm{H}^{ \pm} \rightarrow \tau \nu\right)$ for the L3 experiment. Table 1 gives lower mass limits at $95 \%$ C.L. for the four LEP experiments. A preliminary combined limit of $77.3 \mathrm{GeV} / \mathrm{c}^{2}$ has been derived. 


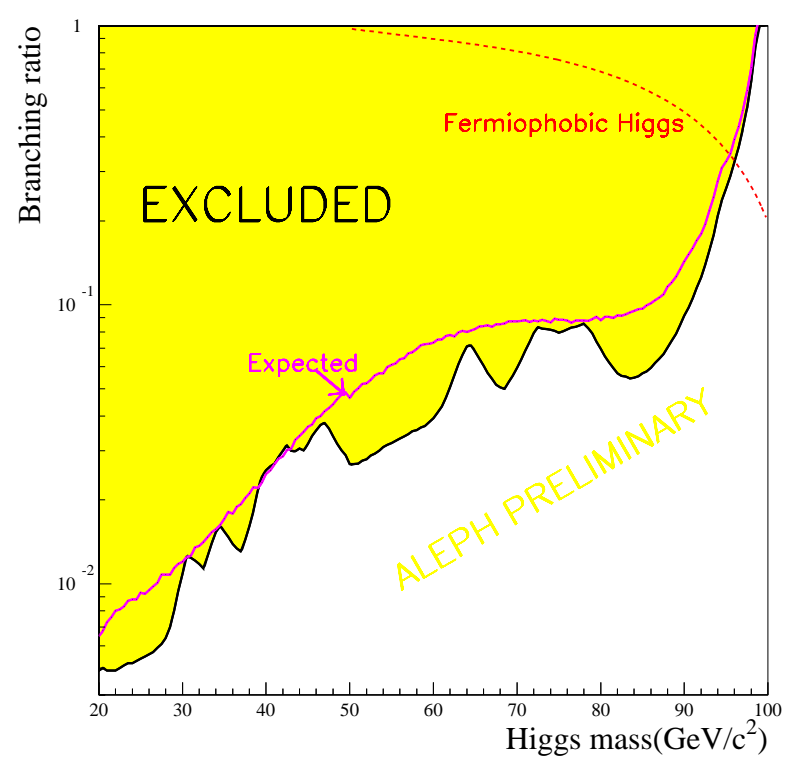

Figure 3. ALEPH 95\% confidence level upper limit on $\mathrm{B}\left(\mathrm{h}^{0} \rightarrow \gamma \gamma\right)$ for a Standard Model Higgs production rate.

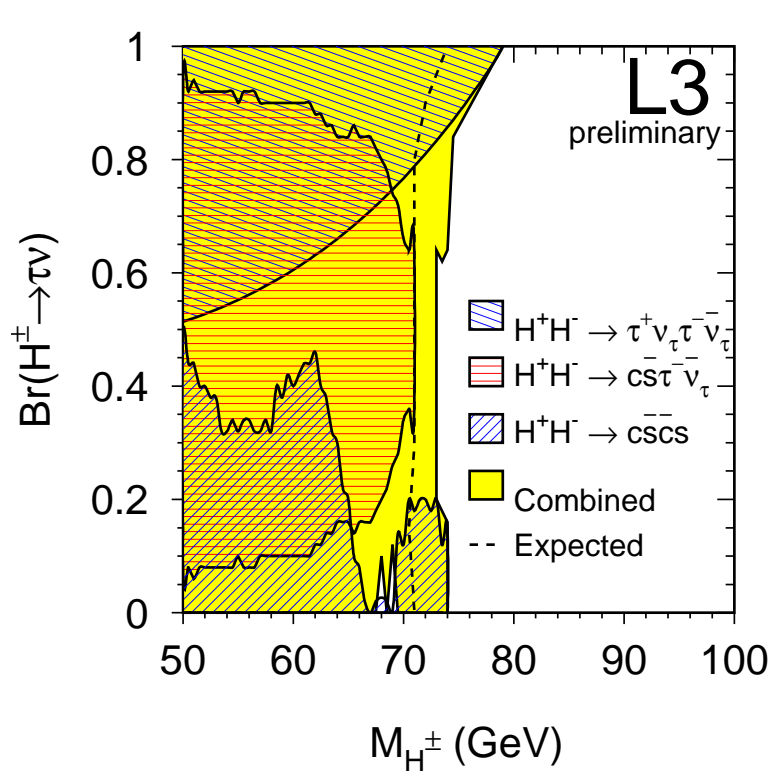

Figure 4. L3 limit at 95\% C.L. on the mass of charged Higgs bosons as function of $\mathrm{B}\left(\mathrm{H}^{ \pm} \rightarrow \tau \nu\right)$.

\section{Future prospects}

At the end of year 2000, the LEP centre-of-mass energy will be increased up to about $205 \mathrm{GeV}$. The invisible Higgs and the fermiophobic Higgs could be excluded up to a mass of $110 \mathrm{GeV} / \mathrm{c}^{2}$, as well as the charged Higgs in the difficult region of the $\mathrm{W}$ mass. At the Tevatron and LHC, the dominant production mechanism for a Standard Model Higgs (gluon fusion) is useless for the invisible Higgs decay (since it leads to gg $\rightarrow$ nothing) and is forbidden for a fermiophobic Higgs. Thus, the dominant process is associated production via quark-antiquark fusion which has between a factor 2 to 10 lower cross section. In the future the exotic Higgs searches will be much more difficult than Standard Model searches. With an integrated luminosity of $100 \mathrm{fb}^{-1}$ LHC experiments should be able to discover an exotic Higgs up to a mass of $1 \mathrm{TeV} / \mathrm{c}^{2}$ if the coupling with gauge bosons is close to the Standard Model coupling.

\section{Conclusions}

New preliminary searches on exotic Higgs decays have been performed by the four LEP experiments and the two Tevatron experiments. No evidence of new phenomena have been observed. New lower limits of the order of $95 \mathrm{GeV} / \mathrm{c}^{2}$ on the invisible Higgs mass and fermiophobic Higgs mass have been set. A charged Higgs is excluded up to a mass of $77.3 \mathrm{GeV} / \mathrm{c}^{2}$. 